\title{
CONDUCTA CORROSIVA DE LOS ALAMBRES DE NITI EN SALIVA ARTIFICIAL Y ENJUAGUES BUCALES CON FLUORUROS
}

\author{
${ }^{1}$ Edwin Ospino Acevedo, ${ }^{2}$ Johnch Mauricio Navarro Carrascal. \\ ${ }^{1}$ Odontólogo U. Javeriana, Estudiante III año Especialización en Ortodoncia U. Santo Tomás. \\ ${ }^{2}$ Odontólogo U. Santo Tomás, Estudiante III año Especialización en Ortodoncia U. Santo Tomás
}

Autor responsable de correspondencia: Edwin Ospino A.

Correo electrónico:edwinospino@gmail.com

\section{RESUMEN}

Objetivo: Establecer si los enjuagues bucales con fluoruros alteran el estado superficial de los alambres titanio y níquel (NiTi) y aceleran su proceso de corrosión.

Materiales y métodos: Se evaluaron 21 arcos de aleación equimolar de titanio y níquel (55,6\% de Ni y 44\% de Ti) de 0,016 pulgadas después de estar en contacto con cada una de la diferentes soluciones (saliva artificial, enjuague con o sin alcohol) en celdas termostatadas a $37^{\circ} \mathrm{C}$ donde siempre permanecieron los elementos protésicos en cada una de las soluciones de prueba, acelerando los procesos de corrosión por medio de técnicas electroquímicas de polarización potenciodinámica, cronoamperometría y espectroscopia de impedancia electroquímica

Resultados: Se obtuvo una mayor velocidad de corrosión en los alambres sometidos a enjuague sin alcohol, comparado con el enjuague con alcohol y saliva artificial respectivamente $(\mathrm{P}<0.05)$. Aunque la solución de saliva artificial presentó una mayor tendencia a corroer el material, la velocidad de corrosión fue menor comparado con los enjuagues.

Conclusión: Los valores bajos de potencial de corrosión mostrados son señales de que el material presenta un pequeño rango de estabilidad y con cualquier cambio de condiciones de $\mathrm{pH}$ o temperatura puede corroerse. La disolución de iones de níquel fue menor a la dosis considerada como tóxica para el cuerpo humano. [Ospino E, Navarro JM. Conducta corrosiva de los alambres de NiTi en saliva artificial y enjuagues bucales con fluoruros. Ustasalud 2009; 8: 83 - 90]

Palabras clave: Alambres NiTi, Enjuagues bucales, Corrosión, Ortodoncia.

\section{CORROSSION BEHAVIOUR OF NITI WIRES IN ARTIFICIAL SALIVA AND FLUORIDE MOUTHWASHES}

\begin{abstract}
Objective: To determine the structural changes and the release of ions from NiTi wires in different solutions, such as artificial saliva and mouthwash with and without alcohol.

Methods: Twenty one arches of nickel-titanium alloy (55,6\% Ni and 44\% Ti), 0,016 inches were evaluated after being in contact with one of the three solutions tested (artificial saliva, mouthwash with and without alcohol) in cells thermostats to $37^{\circ} \mathrm{C}$, speeding up the corrosion process through electrochemical polarization techniques: potentiodinámic, chronoamperometric and Impedance Spectroscopy. Results: It was found a higher rate of corrosion on the wires subjected to mouthwash without alcohol, compared to rinsing with mouthwash with alcohol and artificial saliva, respectively $(\mathrm{P}<0.05)$. Although the artificial saliva presented a greater tendency to corrode equipment, the rate of corrosion was lower compared with the mouthwashes.

Conclusions: The low potential for corrosion signs shown that the material presents a small range of stability and any change in temperature or $\mathrm{pH}$ conditions, can cause them to corrode. The dissolution of nickel ions was less than the considered toxic to the human body.
\end{abstract}

Key words: NiTi wires, Mouthwashes, Orthodontics, Corrosion. 


\section{INTRODUCCIÓN}

Las aleaciones de los metales en los arcos de alambres utilizados en ortodoncia han sido objeto de constante progreso, por esto se consideran como propiedades ideales básicas de estos aditamentos: 1) Superelasticidad, 2) Memoria de forma, 3) Buena resistencia a la corrosión, D) Biocompatibilidad. Los alambres de NiTi (aleación de Níquel y Titanio) son reportados como uno de los alambres que cumplen con estos requerimientos y tienen un excelente desempeño en la rutina ortodóntica diaria.

El principal inconveniente de las aleaciones de NiTi es su alto contenido en Níquel (Ni). Al colocar en contacto este material con en el cuerpo humano, se liberan iones $\mathrm{Ni}$ al medio exterior y a los tejidos circundantes. Aproximadamente un $4 \%$ de los hombres y un promedio de $15 \%$ de las mujeres son alérgicos al Ni. Además, diversos estudios han reportado efectos negativos de los iones Ni sobre los tejidos vivos y algunos mecanismos biológicos. Estos efectos pueden ir desde una leve alergia del paciente hasta la formación de tumores. ${ }^{1}$

Existen características tanto físico-químicas como del comportamiento biológico del material que se estudian sistemáticamente en la evaluación de su biocompatibilidad. Un inventario no exhaustivo de estos parámetros críticos incluye la toxicidad, la composición química, la energía superficial, el tamaño, la forma, la relación peso/superficie (peso específico), la textura de la superficie, las cargas superficiales, las proteínas absorbidas en la superficie, la solubilidad, la porosidad, el tamaño de los poros, la velocidad de degradación, y los productos de degradación (soluciones en contacto y pH). ${ }^{2}$

La inmersión de NiTi en una solución de fluoruro induce una degradación de las propiedades mecánicas del alambre de NiTi a causa de una fragilización de hidrógeno. Este proceso destruye la película protectora de óxido y lleva a una disminución en la resistencia a la corrosión. Según algunos estudios de absorción atómica, la concentración de iones liberados en medios sin células presentes, no es proporcional a la composición de las aleaciones. ${ }^{3}$

A pesar de que el Ni es un elemento que provoca reacciones de toxicidad y alergias importantes, muchos estudios han demostrado que presenta una citocompatibilidad muy similar a la de los aceros inoxidables, y, a veces, incluso a la del Ti puro. ${ }^{4,5}$ Está aceptado que estos buenos resultados se deben a la presencia de una fina capa de óxido de $\mathrm{Ti}\left(\mathrm{TiO}_{2}\right)$ en la superficie del NiTi, que limita la liberación de iones y protege el material de los fenómenos de corrosión. Sin embargo, existen resultados contradic- torios en la literatura, principalmente debido a las distintas condiciones experimentales. ${ }^{6}$

Las aleaciones de níquel estimulan este tipo de respuesta inmune por su entrada a través del tejido conectivo del huésped sobre el contacto directo con la piel o mucosa. Aunque la alergia de la mucosa al metal no es tan común, y en vista de la alta frecuencia de alergia cutánea al níquel, se han reportado pocos casos de reacciones alérgicas orales en la literatura dental. ${ }^{7}$

$\mathrm{Al}$ iniciar el tratamiento de ortodoncia, el profesional en su compromiso con la salud oral escoge el plan de tratamiento ideal y con ello los aditamentos necesarios para la elaboración de dicho tratamiento. Estos aditamentos presentan un común denominador que hace referencia a la aleación de metal y que varía de acuerdo a la necesidad del tratamiento a realizar. ${ }^{4}$ La aleación de NiTi se encuentra presente en la práctica diaria del ortodoncista, y ha sufrido diferentes cambios en su composición de acuerdo con una serie de especificaciones para reforzar sus falencias, apenas imaginables tratándose de un componente ajeno al cuerpo humano y que va durar aproximadamente algunos meses en boca. En este lapso de tiempo serían incontables la cantidad de sustancias con las que entran en contacto estas aleaciones; puede que algunas de ellas sean el detonante para producir alteraciones sobre las superficies de los alambres y desencadenen en algunos individuos problemas en su salud.

Los niveles de flúor en la cavidad oral varían de acuerdo con la calidad de higiene del paciente, es usual ver el constante aumento de productos odontológicos con contenido de flúor. Estas ayudas para mejorar la higiene oral tienen como componente principal los fluoruros y promueven la formación de iones de fluoruro de calcio que se adhieren a los dientes y estimulan su remineralización para protegerlos contra el ataque ácido. Los enjuagues que contienen fluoruro ayudan a controlar el desarrollo de la caries dental y protegen el esmalte. Es fundamental tener en cuenta la condición de este vehículo al contacto con las aplicaciones ortodónticas, y la influencia de estos enjuagues de fluoruro en la corrosión electroquímica de alambres de NiTi. El empeoramiento de la resistencia a la corrosión de los alambres de NiTi tiene dos consecuencias: la primera es la pérdida de las propiedades físicas que juegan un papel importante en el éxito del tratamiento clínico, y la segunda es la liberación de iones de Ni que causa reacciones alérgicas.

El propósito de este estudio fue establecer si los enjuagues bucales con fluoruros alteran el estado superficial de los alambres NiTi y aceleran el pro- 
ceso de corrosión al tener en cuenta la posible acción de los diferentes componentes de los enjuagues utilizados. Además, contribuir al conocimiento básico y fundamental acerca del comportamiento corrosivo de elementos ortodónticos de los alambres de Nitinol.

\section{MATERIALES Y MÉTODOS}

Se realizó un estudio experimental in vitro sobre la influencia de los enjuagues orales con fluoruro en la resistencia a la corrosión de los alambres de NiTi. Se seleccionaron materiales como saliva artificial, dos clases de enjuagues bucales Plax (Colgate ${ }^{\circledR}$ ) con y sin alcohol, 21 arcos de NiTi de calibre 0,016 (Highland $\left.\operatorname{Metal}^{\circledR}\right)$. Se determinó la condición superficial del alambre a través de microscopia tridimensional $\left(\right.$ Hyrox $^{\circledR}$ ) para establecer la presencia de rugosidades e irregularidades del estado superficial de los alambres de NiTi, de acuerdo con estado inicial y final.

Las variables que se tuvieron en cuenta para este estudio fueron: Solución, Peso inicial y Peso final, Potencial de corrosión (Corrosión), Potencial de activación (Corrosión), Potencial de transpasivación (Corrosión), Potencial de activación (Liberación de iones níquel), Potencial de pasivación (Liberación de iones níquel), Potencial de transpasivación (Liberación de iones níquel) y Potencial de pasivación (Tiempo de liberación de iones).

El análisis de la composición química se realizó por la técnica de espectrometría de fluorescencia de rayos X por energía dispersa. Se analizaron tres puntos diferentes y la composición correspondió al promedio de los resultados obtenidos. El equipo utilizado fue un espectrómetro de fluorescencia de rayos $\mathrm{X}$ de energía dispersa Shimadzu EDX $800 \mathrm{HS}$, con un detector de estado sólido $\mathrm{Si}$ (Li) y una fuente de rayos $\mathrm{X}$ de Rodio, con un colimador de $3 \mathrm{~mm}$ sin filtro. La cuantificación de los elementos se realizó con el método de los parámetros fundamentales (FP) mediante el software DXP-700E Versión 1.00 REL. 014.

La caracterización química y metalográfica se basó en la puesta a punto de los equipos y se realizó mediante la calibración de los electrodos de trabajo en los medios fisiológicos empleados con el fin de de- terminar el potencial de estabilidad. Algunos de los equipos empleados fueron:

Potenciostato/galvanostato GAMRY, Electrodos de $\mathrm{Ag} / \mathrm{AgCl}$, Baño termostatado con recirculación y control de temperatura, pH-metro, Espectrofotómetro para determinación cuantitativa por absorbancia de luz, Espectrofotómetro de absorción atómica, Fluoroscopio de rayos X, Microscopio, Conductivímetro.

El montaje experimental de los estudios electroquímicos consistió en un conjunto de recomendaciones tomadas de las normas ASTM y principalmente de la ISO, que regula la evaluación biológica simulada mediante la siguiente norma: ISO 10993-15:2000 parte 15: identificación y cuantificación de productos de degradación de metales y aleaciones. El electrolito debe tener una pureza de grado analítica y debe ser disuelto en dos tipos de aguas: destilada o desionizada, de acuerdo a la norma ISO 3696. Los electrolitos usados pueden ser: solución de cloruro de sodio al $1 \%$, saliva artificial.

El electrolito se renovó para cada experimento, se emplearon $500 \mathrm{ml}$ de solución para cada prueba. Con el fin de realizar la prueba piloto, se evaluaron arcos de aleación de níquel-titanio para determinar los cambios estructurales, la liberación de iones en diferentes medios agresivos y su interacción con las diferentes composiciones de saliva artificial y enjuagues bucales con y sin alcohol (Tabla 1), se evaluó a nivel de laboratorio (in vitro) y en condiciones reales de temperatura, aireación y $\mathrm{pH}$, donde se aceleraron los procesos de corrosión por medio de técnicas electroquímicas de polarización potenciodinámica, cronoamperometría y espectroscopia de impedancia electroquímica, evaluándose luego la morfología superficial y la velocidad de liberación iónica después de ser expuestas a los ensayos electroquímicos.

Se realizó la prueba de polarización potenciodinámica cíclica (Figura 1), con la cual se obtuvieron las pendientes tafel de velocidad de la reacción anódica y catódica y los rangos de potencial de trabajo, para determinar el potencial aplicado para acelerar la corrosión del material en saliva sintética.

Tabla 1. Composición de las soluciones de prueba

\begin{tabular}{ccc}
\hline Saliva artificial & Enjuague bucal con alcohol PLAX & Enjuague bucal sin alcohol PLAX $^{\circledR}$ \\
\hline $\mathrm{KCl} 1,47 \mathrm{~g} / \mathrm{l}$ & $\mathrm{Na}_{2} \mathrm{~F} 0,05 \%$ & $\mathrm{Na}_{2} \mathrm{~F}_{0}, 05 \%$ \\
$\mathrm{NaHCO}_{3} 1,25 \mathrm{~g} / \mathrm{l}$ & Triclosan 0,03\% & Triclosan 0,03\% \\
$\mathrm{KSCN}_{0,52} \mathrm{~g} / \mathrm{l}$ & Alcohol etílico & Alcohol etílico \\
$\mathrm{KH}_{2} \mathrm{PO}_{4} \cdot \mathrm{H}_{2} \mathrm{O} 0,19 \mathrm{~g} / 1$ & Glicerina & $\mathrm{NaOH}$ \\
\hline
\end{tabular}




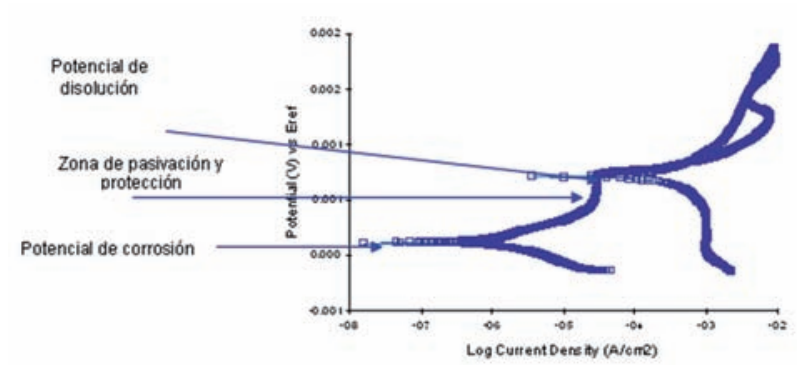

Figura 1. Curva de polarización potenciodinámica cíclica de NiTi en saliva sintética obtenida en un rango de potencial entre $-250 \mathrm{mV}$ y $1400 \mathrm{mV}$

Después de la prueba electroquímica de polarización potenciodinámica se seleccionó un potencial de $700 \mathrm{mV}$ con el fin de realizar la prueba cronoamperométrica para determinar la corriente de disolución del material, en la cual se aplicó este potencial, por debajo del potencial de disolución; ésta se transpasiva, por 10 minutos, hasta la estabilización del sistema. Este potencial representa el estado real de activación y protección en la implantación de este material en la cavidad bucal. Valores por debajo de este potencial son más conservadores del estado natural del alambre implantado y los valores por encima, asumen un estado de deterioro extremo.

Se tomaron muestras de solución en alícuotas de 10 $\mathrm{ml}$ a 5 minutos y a 10 minutos de imponer el potencial, con el fin de determinar por espectroscopía de absorción atómica con un oxidante de oxígeno y un combustible de acetileno, la cantidad de Níquel en $\mathrm{mg} /$ litro disueltos bajo este potencial.

Para determinar el tiempo real de liberación de iones níquel, se emplea la relación de Faraday, la cual relaciona la cantidad de corriente transferida por el sistema, la masa liberada, el número de electrones involucrados y la constante de Faraday.

La masa correspondiente en un año fue determinada al calcular el volumen perdido de material y suponer una cubierta cilíndrica.

La Espectroscopía de Impedancia permitió evaluar el mecanismo de degradación de los materiales y su estabilidad en un medio de prueba. El diagrama obtenido en la técnica potenciodinámica permitió evaluar el comportamiento cinético (velocidad) y termodinámico (potencial), mediante la aplicación de una rampa de velocidad en un rango de potencial en el cual se toma como referencia el potencial de corrosión del sistema. La corrosión en sistemas acuosos involucró transferencia electrónica o de carga en el sistema, pero un cambio de potencial incide en la velocidad de corrosión del sistema. Un metal en solución acuosa, puede reaccionar, ya sea con el oxígeno o con el hidrógeno del agua, reacción anódica o catódica respectivamente, dependiendo del grado de acidez de la solución, una de estas reacciones puede ocurrir más espontáneamente que la otra.

El valor de potencial donde la velocidad de reacción anódica, se iguala con la velocidad de reacción catódica, se llama potencial de corrosión, por debajo de este potencial, suceden las reacciones de la superficie metálica, ya sea con los grupos hidroxilos $(\mathrm{OH}-)$ de la disociación del agua o con los grupos $\mathrm{H}$, potenciales más bajos que el potencial de corrosión. Por encima del potencial de corrosión, la superficie metálica reacciona con el oxígeno del agua y tiende a presentar el estado en el cual se forma una capa de pasivación u óxido estable. Valores de aproximadamente 700 $\mathrm{mV}$ por encima del potencial de corrosión se presenta, transpasivación o picado en algunos materiales (aceros inoxidables o materiales ricos en níquel), el cual es un estado de inestabilidad donde un elemento de la aleación del material, cambia de estado de oxidación y libera una gran cantidad de masa, ${ }^{8}$ un estado de envejecimiento casi crítico del material y condiciones altamente corrosivas. La polarización con otros potenciales puede dar resultados más reales de condiciones normales de corrosividad bucal. Potenciales cerca del potencial de corrosión da como resultado una corrosividad baja, en la zona de pasividad o protección, corrosividad normal o intermedia, en la zona cerca al potencial de transpasivación, alta corrosividad bucal (bajos niveles de $\mathrm{pH}$ o actuación de elementos activos en enjuagues bucales por tratamientos prolongados) y potenciales en el potencial de transpasivación, son condiciones en los cuales la corrosividad bucal es muy alta o el material presenta defectos metalúrgicos de fábrica.

\section{RESULTADOS}

\section{Análisis superficial}

La observación microscópica inicial mostró una superficie rayada pero lisa y continua, sin profundidad como se aprecia en la Figura 2. La superficie del alambre NiTi después de exponerse a las soluciones presentó una superficie rugosa e irregular con profundidad como se observa en la Figura 3.

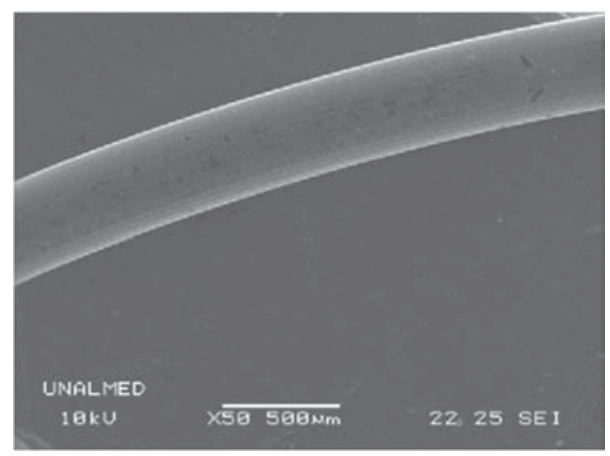

Figura 2. Estado superficial inicial alambre NiTi. 


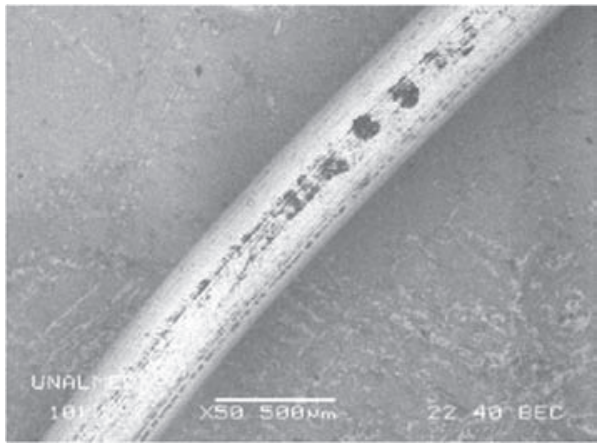

Figura 3. Estado superficial del alambre NiTi después de la prueba

\section{Medida de potencial de corrosión para el NiTi en diferentes soluciones}

Los valores promedio para el potencial de corrosión en los alambres sumergidos en saliva artificial fueron $-0.432 \mathrm{~V} / \mathrm{SCE}$ en el enjuague sin alcohol y $-0,134$ $\mathrm{V} / \mathrm{SCE}$ y para el enjuague con alcohol fue $-0.423 \mathrm{~V} /$ SCE. En la Figura 4 se muestra el potencial contra el tiempo de la prueba realizada.

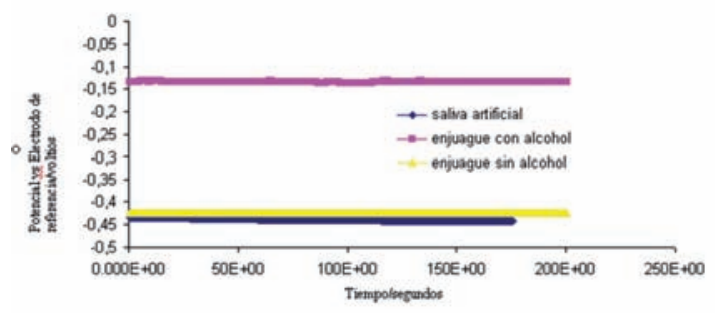

Figura 4. Potencial de circuito abierto de NiTi en saliva artificial. Se observó que en los tres casos, la estabilidad con el tiempo se alcanza inmediatamente. Los valores más negativos de potencial de corrosión se obtuvieron con saliva artificial y con enjuague sin alcohol, y más positivo con enjuague bucal con alcohol, lo que indica que la presencia de alcohol disminuye la tendencia del material a corroerse, tal vez por la formación de grupos funcionales sobre la superficie que inhiben la formación de productos de corrosión inestables que puedan ir a solución en forma iónica.

Se encontraron diferencias estadísticamente significativas en el potencial de corrosión de los alambre NiTi sumergidos en cada uno de los electrolitos utilizados en este estudio $(p=0.000)$. Los valores promedios para el potencial de activación fueron de $-0.17 \mathrm{~V} / \mathrm{SCE}$ para saliva artificial, $0.122 \mathrm{~V} / \mathrm{SCE}$ para enjuague con alcohol, y de $-0.208 \mathrm{~V} / \mathrm{SCE}$ para enjuague sin alcohol y se hallaron diferencias estadísticamente significativas para esta variable $(p=0.000)$.

Los valores promedios para el estado de transpasivación fueron de $0.41 \mathrm{~V} / \mathrm{SCE}$ para saliva artificial, $0.688 \mathrm{~V} / \mathrm{SCE}$ para enjuague con alcohol, y de -0.405
V/SCE para enjuague sin alcohol y se hallaron diferencias estadísticamente significativas para esta variable $(\mathrm{p}=0.000)$.

\section{Medida de liberación de iones en los diferen- tes electrolitos}

Al realizar el análisis de varianza para el estado de activación según la solución, se encontraron diferencias significativas mediante la prueba de ANOVA $(p=00.000)$. Al realizar el análisis de varianza para el estado de pasivación según la solución, se encontraron diferencias significativas mediante la prueba de ANOVA $(p=0.000)$. Al realizar el análisis de varianza para el estado de transpasivación según la solución, no se encontraron diferencias significativas mediante la prueba de ANOVA $(\mathrm{p}=0.0768)$.

\section{Tiempo de liberación de iones}

Al realizar el análisis de varianza para el estado de pasivación (tiempo liberación de iones) según la solución, se encontraron diferencias significativas mediante la prueba de ANOVA $(\mathrm{p}=0.000)$.

\section{Espectroscopía de Impedancia}

En la Figura 5 se presenta el espectro de Bode de Impedancia para las muestras de Nitinol experimentadas en saliva artificial, enjuague bucal sin y con alcohol.

La muestra en saliva artificial presenta mayor resistencia a la corrosión pero es casi similar a la muestra con enjuague bucal con alcohol. La menor resistencia a la corrosión la presentó la muestra con enjuague sin alcohol. Esto se observa en la Figura 6.

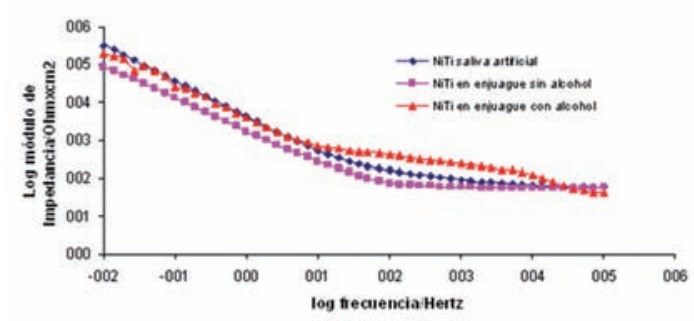

Figura 5. Impedancia para aleación NiTi en saliva artificial, enjuague bucal con alcohol y sin alcohol.

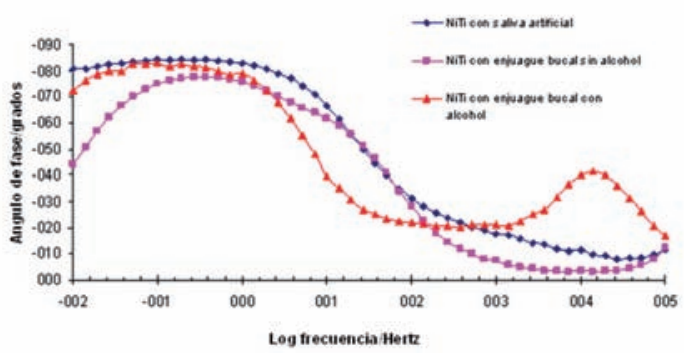

Figura 6. Resistencia a la corrosión de las 3 soluciones. 


\section{Pérdida de peso alambre}

El promedio inicial para los alambres sumergidos en saliva artificial era de 0.13854 gr y el promedio después de finalizada la prueba era de 0.13152 gr.

El promedio inicial para los alambres sumergidos en enjuague bucal con alcohol era de 0.13900 gr y el promedio después de finalizada la prueba fue de 0.13553 gr. El promedio inicial para los alambres sumergidos en enjuague bucal con alcohol era de 0.13315 gr y el promedio después de finalizada la prueba fue de $0.13353 \mathrm{gr}$.

\section{DISCUSIÓN}

Aunque varios estudios han demostrado que los arcos de alambre de aleaciones de NiTi poseen una buena biocompatibilidad, su alto contenido de níquel y la posible disolución durante la corrosión aún son motivo de preocupación. Las reacciones alérgicas observadas en pacientes con ortodoncia sensibilizados con el níquel (han mantenido contacto previo con aleaciones que contienen níquel), son producidas porque el níquel causa una proliferación de linfocitos, lo cual puede producir reacciones alérgicas cuando vuelven a ser receptores de elementos con níquel.

La International Agency for Research on Cancer (IARC) ha establecido que el $\mathrm{Ni}$ metálico es un posible agente cancerígeno, sin embargo no son completas las evidencias clínicas que avalen este argumento. ${ }^{9}$ Sahmali y Kural estudiaron los efectos sistémicos de aleaciones dentales que contenían níquel. Los animales experimentalmente sensibilizados al níquel mostraron niveles significativamente mayores de níquel en el suero que los animales controles. Los niveles de níquel tanto en los animales experimentales como en los controles se incrementaron con el tiempo. ${ }^{10}$

Asimismo, Arikan manifiestó que no se conoce si el uso intraoral de aleaciones de níquel puede causar algún tipo de reacción sensibilización. Este autor realizó un estudio sobre 10 pacientes para determinar si dichas aleaciones podrían producir cambios en las células sanguíneas (linfocitos, T-Iinfocitos, neutrófilos, eosinófilos, basófilos y monocitos). Los resultados mostraron que no hay cambios significativos en las poblaciones de T-Iinfocitos y monocitos. Pero sí se encuentra una disminución de eosinófilos circulantes y un aumento en las poblaciones de neutrófilos y basófilos circulantes. ${ }^{11}$

En este estudio los valores de níquel liberado en el alambre al estar en contacto con enjuagues bucales, con y sin alcohol, fueron de $2,89 \mathrm{mg} /$ año y 2,22 $\mathrm{mg} / \mathrm{año}$, respectivamente; se encuentran por debajo de los valores máximos tolerados por el cuerpo hu- mano, cabe anotar que estos datos se registraron en condiciones bastante controladas y con arcos de alambre con mínima manipulación, libres de cualquier tipo de fricción y sin deformaciones por dobleces del alambre. Esto concuerda con Venugopalan quien reportó una liberación de níquel en alambres NiTi muy por debajo de los niveles permisibles en la ingesta de la dieta humana, estos valores se encontraban en niveles no detectables en los primeros días tras la inmersión en un medio fisiológico. ${ }^{12}$ Shahrabi y colaboradores reportaron que la agresividad relacionada con cambios en la temperatura, $\mathrm{pH}$ y presencia de otros metales causaban corrosión galvánica por la diferencia de potenciales y rozamiento, esto podría causar una gran cantidad de pérdida de masa que en muchos casos era difícil de cuantificar y hace que el proceso de corrosión se acelere hasta 20 veces más de lo normal. Además de la permeabilidad de la capa, la presencia de defectos, dobleces, ralladuras o una combinación de éstos, eran sitios muy activos de corrosión, que aumentan varias veces la velocidad de liberación de níquel, así el material en la mayor parte del área esté en el estado de corrosión general. ${ }^{13}$

Algunos autores han reportado que las aleaciones de NiTi exhiben en la agresividad de los fluidos simulados un comportamiento completamente satisfactorio acerca de la corrosión localizada caracterizado por la presencia de una amplia gama de pasividad a valores altos de potencial. El trabajo de Oshida y colaboradores evidenció bajos valores de potencial al picado y numerosas oscilaciones de corriente pasiva anódica por potenciales de electrodos más bajos que $500 \mathrm{mV}^{14}$ Estas oscilaciones son atribuidas por la exposición a un medio agresivo y áreas de superficie insuficientemente protegidas por la película pasiva que pueden ser sujetas a repasivaciones repentinas.

En las tres soluciones utilizadas en este estudio, la estabilidad con el tiempo alcanzó inmediatamente, los valores más negativos de potencial de corrosión que se obtuvieron con saliva artificial y con enjuague sin alcohol; en el caso del enjuague bucal con alcohol, este potencial fue más positivo e indica que la presencia de alcohol disminuye la tendencia del material a corroerse, tal vez por la formación de grupos funcionales sobre la superficie que inhiben la formación de productos de corrosión inestables que puedan ir a solución en forma iónica.

De acuerdo con algunos estudios, la saliva puede contener $500 \mathrm{mg} / \mathrm{L}$ de iones cloruro, suficientes para inducir transformaciones corrosivas en las estructuras de NiTi. Esto concuerda con los resultados obtenidos en este estudio después de la inmersión 
de los alambres NiTi en saliva artificial como se puede observar en la Figura 7. Es bien sabido que la resistencia a la corrosión de la aleación NiTi puede ser alterada por el efecto de los iones $\mathrm{Cl}$ - sobre el estado pasivo natural del NiTi, lo que crea un estado de transpasivación o corrosión localizada, que puede aumentarse en ambientes agresivos, propiedad llamada: susceptibilidad a la corrosión localizada. ${ }^{15}$

Las pequeñas variaciones en el $\mathrm{pH}$ local, debido a los procesos propios de estabilización termodinámica del material con el medio, y a los procesos biológicos y químicos variantes, pueden formar y conducir a la acción del $\mathrm{HCl}$, bajo alguna concentración y temperatura. ${ }^{15}$ Este registro podría ser controversial, ya que se esperaba una menor tendencia a la corrosión en los alambres NiTi al estar en contacto con enjuagues bucales libres de bases alcohólicas (Figura 8). Este concepto podría estar a la mano, a la hora de recomendar un enjuague bucal al paciente con tratamiento ortodóntico. En el caso de los alambres de NiTi, en el estado de corrosión general (condiciones normales de funcionamiento), se forma una capa de óxido compacta de óxido de titanio $\left(\mathrm{TiO}_{2}\right)$, que protege al material y logra disminuir la velocidad de liberación de níquel al organismo. Sin embargo, los dos procesos de corrosión son distinguibles. Esta capa no es del todo impermeable, a través de ella ocurre la migración iónica hacia la superficie a una velocidad que depende de la agresividad del medio fluidico que la contiene. ${ }^{16}$

En las pruebas de impedancia, la muestra en saliva artificial presenta mayor resistencia a la corrosión pero es casi similar a la muestra con enjuague bucal con alcohol. La menor resistencia a la corrosión la presentó la muestra con enjuague sin alcohol. Es importante observar en el rango de medias frecuencias en la muestra con alcohol, en la cual se presentó una variación en el comportamiento, diferente de las otras en saliva y con enjuague sin alcohol relacionado con la formación de un producto de corrosión estable, el cual puede inhibir o disminuir la velocidad de corrosión del material (Figura 9).

Un aumento en el ángulo de fase entre $10.000 \mathrm{y}$ $30.000 \mathrm{Ohmxcm}$, confirma este comportamiento. En el caso de los alambres de NiTi, en el estado de corrosión general (condiciones normales de funcionamiento), se forma una capa de óxido compacta de óxido de titanio $\left(\mathrm{TiO}_{2}\right)$, la cual protege al material y logra disminuir la velocidad de liberación de níquel al organismo, sin embargo, dos procesos de corrosión son distinguibles. ${ }^{17}$ Esta capa no es del todo impermeable, a través de ella ocurre migración iónica hacia la superficie a una velocidad que depende de la agresividad del medio fluidico que la contiene.
Schiff y colaboradores han demostrado que los diferentes tipos de enjuagues bucales pueden alterar de manera inconmensurable el grado de corrosión y la superficie del alambre de NiTi, recomienan el uso de enjuagues bucales que puedan tener menos efectos adversos sobre la integridad de la aleación. ${ }^{18}$

El peso obtenido después de la prueba no es un indicativo confiable de la pérdida de iones en solución ya que el material puede ganar masa por la formación del óxido y perderla a través de éste, por lo tanto, se requiere de un análisis electroquímico para separar estas contribuciones a la pérdida y ganancia de masa. Es por esto que los valores promedios de pérdida de peso para los alambres de NiTi inmersos en enjuague bucal sin alcohol presentan un promedio mayor en el peso final comparado con el inicial.

Podría ser conveniente realizar procesos de electropulido y anodización de los alambres de NiTi para reducir significativamente la disolución del níquel en los alambres de NiTi debido a la formación de una resistente capa superficial de óxido de titanio. ${ }^{18,19}$

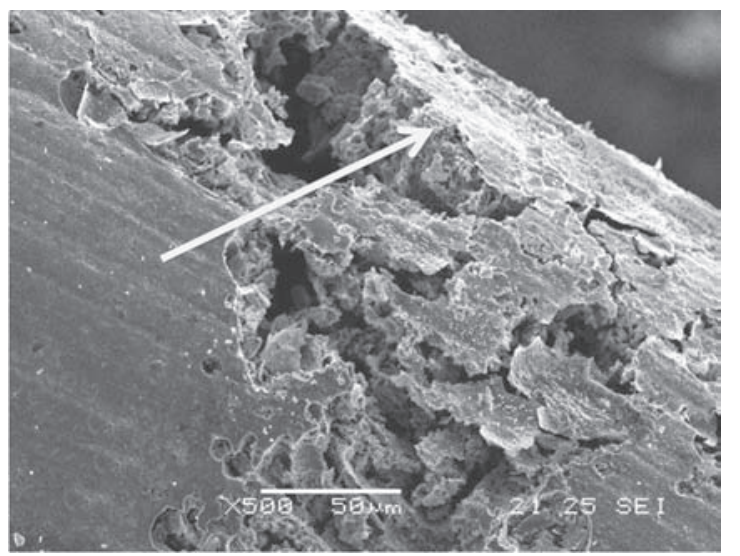

Figura 7. Estado superficial del alambre NiTi después de la prueba en saliva artificial. Micrografia a X500

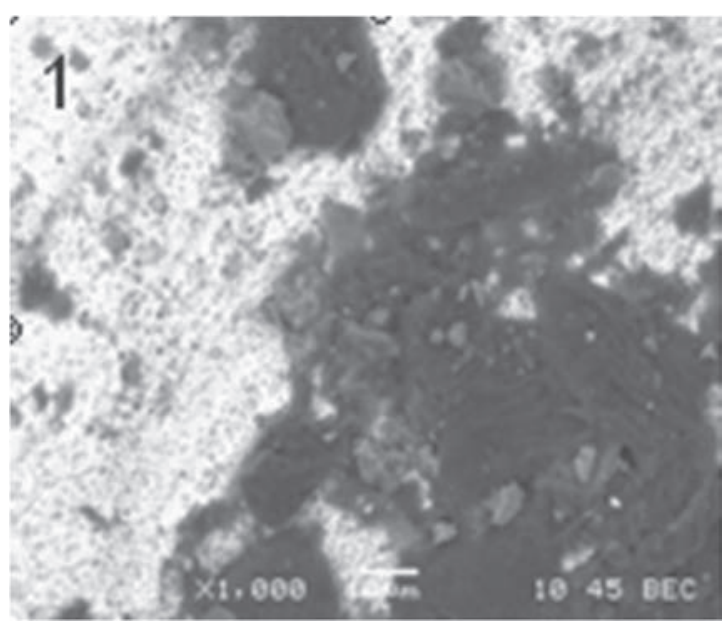

Figura 8. Estado superficial del alambre NiTi después de la prueba enjuague bucal sin alcohol. Micrografia a X500. 


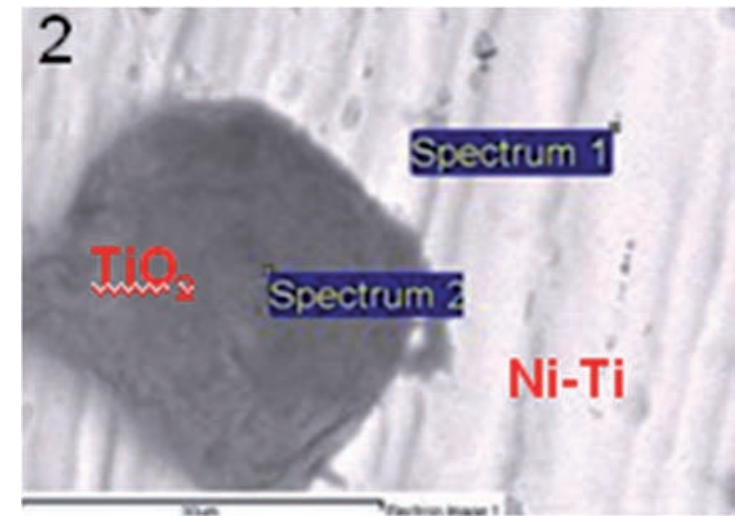

Figura 9. Estado superficial del alambre NiTi después de la prueba enjuague bucal con alcohol. Micrografia a X500.

\section{Conclusiones}

Se obtuvo una mayor velocidad de corrosión en los alambres expuestos a enjuague sin alcohol, comparado con los enjuagues con alcohol y saliva artificial, respectivamente.

Aunque la solución de saliva artificial presentó una mayor tendencia a corroer el material, la velocidad de corrosión fue menor comparada con los enjuagues. Sin embargo, los valores bajos de potencial de corrosión mostrados, son señales de que el material presenta un pequeño rango de estabilidad y con cualquier cambio de condiciones de $\mathrm{pH}$ o temperatura, puede corroerse.

Es claro que en los diagramas de los espectros de Bode se presentó un comportamiento inusual en las muestras probadas con enjuagues con alcohol que demostró que se pueden formar compuestos estables en la superficie que retarden el deterioro o liberación de iones en el material.

Es fundamental complementar este estudio in vitro, con un estudio in vivo, donde se puedan aplicar los resultados obtenidos primariamente en esta investigación y compararlo con las situaciones reales presentadas en la cavidad oral. Adicionalmente, sería interesante realizar investigaciones sobre alambres NiTi anodizados para saber si ofrecen una mayor resistencia a la corrosión que los alambres NiTi convencionales.

\section{BIBLIOGRAFÍA}

1. Kao C, Ding S, He H. Cytotoxicity of orthodontic wire corroded in fluoride solution in vitro. Angle Orthod 2007; 77: 349 - 354.

2. Rocher P, Medawar L. Biocorrosion and cytocompatibility assessment of NiTi shape memory alloys. Scripta Materialia 2004; 50: 255 - 260.

3. Schiff N, Grosgogeat B, Lissac M, Dalard F. The influence of fluoride ions on the corrosion resistance of titanium and NiTi in an artificial saliva and mouthwash. European Cells And Materials 2003; 5: 56 - 57.
4. Bishara SE, Barrett RD, Selim MI. Biodegradation of orthodontic appliances. Part II, Changes in the blood level of nickel. Am J Orthod Dentofac Orthop 1993; 103: 115 - 119 .

5. Barret RD, Bishar SE, Quirin JK. Biodegradation of orthodontic appliances. Part 1. Biodegradation of nickel and chromium in vitro. Am J Orthod Dentofac Orthop 1993; 103: 8 - 14 .

6. Eliades, T, Athanasiou, E. In vivo aging of orthodontic alloys: Implications for corrosion potential, nickel release and biocompatibility. Angle Orthod 2002; 72: $222-237$.

7. Janson GR, Dainesi EA, Consolaro A, Woodside DG, De Freitas MR. Nickel hypersensitivity reaction before, during, and after orthodontic therapy. Am J Orthod Dentofac Orthop 1998; 113: 655 - 660.

8. Cheng F. Nature of oxide layer formed on NiTi by anodic oxidation in methanol. Materials Letters 2005; 59: 1516- 1520 .

9. Venugopalan R, Trépanier C. Assessing the corrosion behavior of nitinol for minimally-invase device design. Min Invas Ther \& Allied Technol 2000; 9: 67 - 64 .

10. Sahmali SM, Kural O. Systemic effects of nickel-containing dental alloys: analysis of nickel levels in serum, liver, kidney and oral mucosa of guinea pigs. Quintessence Int 1991; 22: 961 - 966.

11. Arikan A. Effects of nickel-chrome dental alloys used in dentistry on saliva and serum nickel levels, peripheral t lymphocytes and some other blood parameters. J Oral Rehabil 1992; 19: 343 - 352.

12. Venugopalan R. Corrosion testing of stents: a novel fixture to hold entire device in deployed form and finish. J Biomed Mater Rs 1999; 48: 829 - 832.

13. Shahrabi T, Sanjabi S, Saebnoori E, Barber ZH. Extremely high pitting resistance of NiTi shape memory alloy thin film in simulated body fluids. Materials Letters 2008; 62: $2791-2794$.

14. Oshida Y, Sachdeva RCL, Miyazaki S. Microanalytical characterization and surface modification of TiNi orthodontic archwires.Biomed Mater Eng. 1992;2:51-69.

15. Matos de Souza R, Macedo de Menezes I. Nickel, chromium and iron levels in the saliva of patients simulated fixed orthodontic appliances. Angle Orthod 2008; 78: $345-350$.

16. Her-Hsiung $\mathrm{H}$, Yu-Hui $\mathrm{H}$. Ion release from NiTi orthodontic wires in saliva with various acidities. Biomaterials 2003; 24: 3585 - 3592.

17. Shabalovskaya SA. Surface, corrosion and biocompatibility aspects of nitinol as an implant material. Biomed Mater Eng 2002; 12: 69 - 109.

18. Wever DJ, Veldhuizen AG, de Vries J, Busscher HJ, Uges DR, van Horn JR. Electrochemical and surface characterization of a nickel-titanium alloy. Biomaterials 1998; 19: 761 - 769 .

19. Jones DA. Principles and Prevention of Corrosion. 2nd. Ed. Upper Saddle River, NY: Prentice Hall. 1996. p. 168 $-198$. 